\title{
Knowledge and health promoting lifestyle behaviors about menopause among perimenopausal female teachers
}

\author{
Samar Alotaibi ${ }^{1}$, Sahar Mohammed Hassan Yakout ${ }^{* 2}$, Salma M. Moawad ${ }^{3}$ \\ ${ }^{1}$ Nursing Department, College of Applied Medical Sciences, Shaqra University, Al Dawadmi, Saudi Arabia \\ ${ }^{2}$ Maternity and Gynaecologic Nursing, Alexandria University, Alexandria, Egypt \\ ${ }^{3}$ Department of Maternity and Child Health Nursing, King Saud University, Riyadh, Saudi Arabia
}

Received: November 28, 2018

Accepted: February 13, 2019

Online Published: March 26, 2019

DOI: $10.5430 /$ jnep.v9n6p113

URL: https://doi.org/10.5430/jnep.v9n6p113

\begin{abstract}
Menopause is a critical period in a woman's life that not only marks the end of reproductive ability, but is also associated with multiple physical, vasomotor, psychological, and sexual complaints. Many of the serious complications that women face during menopause are caused by their own poor lifestyles and their lack of knowledge about self-care. The objective of this study was to assess knowledge and health promoting behavior at menopause among perimenopausal female teachers in Dawadmi City. The study applied cross-sectional descriptive design with stratified random sampling to obtain 348 female teachers statistically calculated from the total of 3,708 teachers. Two tools were used for data collection, Tool I: A structured closed ended questionnaire consisting of two parts for collecting data about sociodemographic information and questions to assess knowledge of perimenopausal female teachers about the menopausal symptoms. Tool II: The Health-Promoting Lifestyle Profile II to measures health-promoting behaviors. Result: $58.9 \%$ of the subjects had poor knowledge, $30.7 \%$ had moderate knowledge and only $10.3 \%$ of teachers had Good and Excellent knowledge levels. Total mean score related to Health Promoting Behaviors was $130.05 \pm 27.555$ which is considered medium in general. On the whole, socio-demographic variable of female teachers did not show significant correlations by using Pearson Chi-Square test and ANOVA test. It also did not show significant correlations for the total knowledge level. However, total behaviors showed a significant correlation to age $(\mathrm{F}=1.358, p=.028)$ and weight $(\mathrm{F}$ $=1.303, p=.049$ ) in the last year category. In conclusion, assessing the knowledge of women about menopause plays an essential role in the development of women's health during menopausal years. Results suggest that participants do not consistently include health-promoting behaviors into their lifestyle, especially physical activity. Therefore, certain health promoting programs and policies should be designed and conducted to promote healthy behavior, in addition to developing processes to promote worksite wellness programs, and to support behavioral changes intended to promote healthy lifestyles.
\end{abstract}

Key Words: Knowledge, Behavior, Health promoting life style, Perimenopausal

\section{INTRODUCTION}

The average life expectancy is forecasted to be 35.98 years between 40-60 years at midlife, and the middle-aged women population is projected to grow rapidly. Middle-aged women's wellbeing has been underscored. During the transition phase of women from midlife to elder status they are

\footnotetext{
*Correspondence: Sahar Mohammed Hassan Yakout; Email: yakoutsahar353@gmail.com; Address: Maternity and Gynaecologic Nursing, Alexandria University, Alexandria, Egypt.
} 
facing a diffrent health issues that decrease quality of their life. ${ }^{[1]}$ Menopause is defined as the permanent cessation of menses for 12 months or more due to cessation of ovarian hormone production. ${ }^{[2]}$ Menopause signals a transition from the reproductive stage to the non-reproductive stage in a woman's life. The common climacteric symptoms experienced by them can be grouped into: vasomotor, somatic, psychological or sexual complaints. It was also noted in some postmenopausal women with long term estrogen deficiency, changes to the cardiovascular or bone which leads to osteoporosis has been established. ${ }^{[3]}$

According to the World Health Organization (WHO), 70\%$80 \%$ of deaths in developed countries and $50 \%-60 \%$ in developing countries are related to lifestyle. ${ }^{[4]}$ In perimenopausal women, health problems such as overweight, cancer, smoking, addiction and cardiovascular diseases are related to changes in lifestyle. Therefore, health promoting behaviors with an emphasis on promoting a healthy lifestyle would increase health, quality of life and reduce costs of treatment. ${ }^{[5]}$

Recent research reported that, in Saudi Arabia, about 919,527 women are at menopausal age of 45-54 years old. ${ }^{[6]}$ Moreover, there is a change in the pattern from communicable diseases to non-communicable diseases in Saudi Arabia. ${ }^{[7]}$ There has been an alarming increase in the prevalence of chronic diseases such as diabetes, hypertension, heart diseases, cancer, obesity and blood disorders. ${ }^{[7]}$ Treatment of chronic diseases is costly and may even be ineffective. So, early prevention is the most effective way to reduce the prevalence of chronic diseases and the costs and difficulties associated with treatment in the later stages of disease. ${ }^{[8,9]}$

Today, improving lifestyle and promoting health are necessary requirements in human society. ${ }^{[8]}$ Health promotion is the science and art of lifestyle change in order to reach desirable goals and achieve a healthy lifestyle. ${ }^{[10,11]}$ Also, the more knowledge one has about menopause, the more they are empowered to cope with menopausal changes. It has been suggested that lack of knowledge regarding menopause makes women more anxious when it is time to deal with menopause and this has negative effects on their emotional state. Changing women's perceptions of menopause by increasing their knowledge related to menopause may cause less emotional disturbance. ${ }^{[10]}$

\subsection{Significance of the study}

Women during the menopause period experience certain physical and psychological changes and face various illnesses such as urogenital, psychological-social, cardiovascular and neurological problems, etc. ${ }^{[1]}$ These issues not only cause great distress and disability for the person but also burdens health institutions with more cost. ${ }^{[12]}$ Familiarity with these changes and understanding their reasons are essential in the life of all women, and helps them enter this stage of their life with adequate knowledge and a healthy lifestyle. ${ }^{[11]}$ Unfortunately, policies and programs of women's health promotion are limited to specific issues such as pregnancy and family planning, and not enough attention is paid to other women's health needs, including the problems of menopausal transition period. So, women need special attention to help them maintain their life and such research will help healthcare provides to obtain necessary data to devise interventions specific to the needs of premenopausal women as well as to identify the areas of self-care deficit.

\subsection{Aim of the study}

The aim of this study is to assess knowledge and health promoting llifestyle behaviors about menopause among perimenopausal female teachers at Dawadmi City.

\subsection{Research objectives}

- Asses the level of knowledge about menopausal period among midlife female teachers.

- Assess the health promoting behavior of midlife female teachers about menopause.

- To examine association between socio- demographic characteristics of the teachers and their level of knowledge and promoting behaviors about menopause.

\subsection{Research questions}

- What is the level of knowledge about menopause among female teachers?

- What are the health promoting behaviors of female teachers about menopause?

- Is there a significant association between sociodemographic characteristics of the teachers and their level of knowledge and health promoting behaviors about menopause?

\subsection{Conceptual definition}

Health Promoting Behavior(HPB): Health promoting behavior is a multidimensional pattern that Pender in 1996 divided into 6 dimensions of nutrition, physical activity, spiritual growth, health responsibility, stress management, and interpersonal relations. ${ }^{[5]}$

Menopause: The permanent cessation of ovarian function and the end of a woman's reproductive potential. ${ }^{[13]}$

Perimenopausal period: The entire reproductive period up to the final menstrual cycle. It is best defined as a time of 
"normal" reproductive function in a woman. ${ }^{[14]}$ In this research, the researcher includes women between 35 and less than 55 years.

Dawadmi city: It is located in the central area of Saudi Arabia. It is about 300 kilometers west of Riyadh. It is a town in Riyadh Province, Saudi Arabia. As of the 2017 census it had a population of 78,034 people.

\subsection{Theoretical background of the study}

Dr. Nola Pender's Health Promotion Model was first coined in 1982 and later revised in 1996 as a "complementary counterpart to models of health protection". Health is defined as a positive self-motivated state rather than only the absence of disease. ${ }^{[17]}$ Health is pursued by a multidimensional nature of people as they cooperate within their environment. Pender describes in this model that health is a lifestyle accustomed by the amount of choices made by the distinct person to essentially live a healthy lifestyle. It was her background in nursing, human development, experimental psychology and education that led her to use a holistic nursing perspective, social psychology and learning theory as the foundations for the Health Promotion Model. ${ }^{[15]}$

\section{MethodS}

\subsection{Research design}

A descriptive cross-sectional design was used to conduct of the study, because the study intends to describe the knowledge and heath promoting behaviors among perimenopausal teachers at Dawadmi city and to find association between the variables.

\subsection{Study setting}

The study was conducted at 14 governmental secondary schools representing the eight educational zones in Dawadmi City. It is located in the central area of Saudi Arabia. It is about 300 kilometers west of Riyadh. There are $375 \mathrm{fe}-$ male schools, and 3,708 teachers at Dawadmi working in these schools according to the last statistics from Ministry of education in 2017.

\subsection{Sampling}

Multistage sampling technique was used as follows:

Stage I: Dawadmi city is divided into 5 sectors: north, south, east, west, and middle of the city. The governmental secondary schools for females in Middle of Dawadmi city was selected randomly.

Stage II: Middle of Dawadmi comprises 8 areas and the total number of governmental female secondary schools is 14 schools distributed among those 8 areas. Six secondary schools were chosen by simple random sampling technique.
Stage III: 58-59 teachers were selected systematically from each school and 348 teachers who fulfill the criteria were involved in the study.

\subsection{Study subjects}

A non-probability, convenience subject of 348 female teachers. The subject consists of (348) female teachers at Dawadmi city. Statistically calculated from the total of 3,708 teachers based on the last census by the Ministry of Education of Dawadmi region. The subject size was determined using the calculated formula:

$\mathrm{SS}=\left(\mathrm{Z}^{2 *}(\mathrm{p})^{*}(1-\mathrm{p})\right) / \mathrm{c}^{2}$ Where:

$\mathrm{Z}=\mathrm{Z}$ value (e.g. 1.96 for $95 \%$ confidence level);

$\mathrm{p}=$ percentage picking a choice, expressed as decimal $(.5$ used for sample size needed);

$\mathrm{c}=$ confidence interval, expressed as decimal. ${ }^{[16]}$

The following criteria will comprise the subjects of this study:

\subsubsection{Inclusion criteria}

Governmental School teacher.

Saudi Nationality.

Teacher aged between 40 to 55 years.

\subsubsection{Exclusion criteria}

Women who had undergone total hysterectomy for any reasons.

\subsection{Tools of data collection}

Tool I: A structured questionnaire sheet was used to collect the data, the researcher developed questionnaire after reviewing the literature. ${ }^{[13,19]}$

It included the following parts:

Part (1): The socio-demographic, obstetric, and medical variables including the women's age, marital status, education level, family income, parity, age of menarche... (about 11 closed questions)

Part (2): Knowledge about menopause among female teachers. It consists of three categories: First category: Measuring knowledge of female teachers about the menopausal period. Second category: Measuring knowledge of female teachers about menopausal symptoms. Third category: Measuring knowledge of female teachers about strategies applied to reduce these symptoms, for example physical problems such as doing physical exercises, then questions about nutrition and so on.

Scoring system: For determining the knowledge level, answers to the 48-multiple choice questions related to knowl- 
edge are scored and summed for each case. For each question, the score is assigned according to the correctness of the answer. The score is 0 (zero) for the wrong or incomplete answer and 1 (one) for the right and complete answer. In order to answer this question, four levels of knowledge were identified (poor, moderate, good and excellent), where the poor level was less than 28.8 points, moderate ranged between 28.9-38.4, while the good ranged between 38.5-43.2, and excellent more than 43.3 points. Herein, the points calculated as well as a score = one, to "correct answer" and a score = zero corresponded to "incorrect answer". Therefore, overall, the maximum and minimum possible scores were 48 and 0 , respectively.

Tool II: Health Promoting Lifestyle Profile II (HPLP II). This instrument was designed by Walker, Sechrist and Pender in 1987 to measure health promoting behaviors. ${ }^{[18]} 52$ health promoting lifestyle behaviors are measured in a fourpoint Likert scale for each item ranging from 1 (never) to 4 (routinely). The HPLP II includes six sub-scales: health responsibility ( 9 items), physical activity ( 8 items), nutrition (9 items), spiritual growth (9 items), interpersonal relationships (9 items), and stress management (8 items).

Scoring: The overall score of the instrument ranges from 52 to 208 , where each domain has its own separate score and higher scores indicate a better adherence to health promoting behavior. In this way, scores 52-103 means a low level of adherence to these behaviors and scores 104-155 and score above 156 imply a moderate and high level, respectively. ${ }^{[19]}$

\subsection{Pilot study}

A pilot study was conducted on 35 participants from the female teachers $(10 \%$ of the total subject). It was done to evaluate the applicability and clarity of the tools, assesse the feasibility of field work, and test the reliability of the questionnaire. The pilot study sample was excluded from the study sample. In light of the result of experiences from the pilot study the necessary modification was made to the questionnaire.

\subsection{Validity}

The tool was reviewed by a statistician, two experts from the Maternal and Gynecological Nursing Department and one expert in the Community Nursing Department. Feedback from the experts led to some item revision, modification or deletion. All the modifications were done.

\subsection{Reliability}

Reliability and validity statistics were obtained using Cronbach's alpha. The reliability is $94.6 \%(p=.000)$ while the validity coefficient is $97.2 \%$. These results were obtained from the Intraclass Correlation Coefficient.

Reliability $=94.6 \%$ was calculated from the questionnaire excluding demographic data.

Cronbach alpha for all questions is 0.902 ; hence, the questionnaire is likely to all be measuring the same construct. Deleting any question did not increase the Cronbach reliability coefficient. Accordingly, there was no need to exclude or modify any question of the questionnaire. In conclusion, as the reliability coefficient for the 48 questions is greater than 0.7 , thus all questions could be considered as a reliable tool to measure the knowledge.

Cronbach's alpha for all questions is 0.953 ; hence, the questionnaire is likely to all be measuring the same construct and can be considered as a reliable measure to determine health promoting lifestyle behaviors for female teachers; as the Cronbach's alpha is greater than 0.7 .

\subsection{Administrative design}

An official letter of permission to conduct the study was obtained from King Saud University, College of Nursing. A data was fielded. An official letter was sent from King Saud University, Nursing College to the Education Administration in Dawadmi city to give permission to collect data. Permission was obtained from the Education Administration in Dawadmi city to collect the necessary data from female teachers in Dawadmi city. The process of approval had taken about three months.

An official letter was sent from King Saud University, Nursing College to the ministry of justice to provide statistical evidence that shows the number of female teachers who were working in schools in Dawadmi city. The ministry responded by sending a document showing the number of teachers.

\subsection{Ethical consideration}

Approval from the Ministry of Education was taken before data collection started. Approval of the participants were obtained in written form before data collection explaining the aim of the study and stating that all information taken from participants in the study will remain strictly anonymous and confidential.

\subsection{Data collection procedure}

An official letter from King Saud University, Nursing College was obtained to be submitted to the administrators at the nominated setting for permission to conduct the study. In order to reach Saudi female teachers at government schools in Dawadmi city, the statistic department in the Ministry of Education was contacted. Eligible subjects based on the December 2016 Ministry of Education statistics were identified 
according to the inclusion criteria. A representative sample of 348 was recruited from the previously detailed sampling technique. The schedules for each school were obtained to determine the suitable time for data collection. Appointment where schedules were arranged for each school based on the appropriate time for them. Meetings were conducted with school managers to ensure their cooperation and to gain their support after taking consent. Data collection takes four days per week to complete. Teachers were met on the day of their appointments, and those who fulfilled the criteria were included in the study. Questionnaires were distributed to available teachers after explaining the purpose of the study and time was allocated for them to seek any clarification. It took around 30 minutes to complete the questionnaires. After collecting completed questionnaires from the teachers, the researcher performed checks and excluded incomplete questionnaires. Data were collected during the period of four months from 20th September 2017 to 20th December 2017.

\subsection{Data analysis}

Data was categorized, coded and analysed according to appropriate statistical methods by using Statistical Package for Social Sciences, SPSS v. 25 (SPSS Inc, IBM). Descriptive statistics was employed to describe the variable frequencies of the sample, mean and standard deviation, represented by tables and figures. Nomograms with confidence intervals (95\%) were established for parameters of the study; $p$-value was significant at $<.05$. Chi-squared test was used to examine the distribution of the data, and ANOVA to compare between variables.

\section{RESUlts}

Table 1 shows the percentage distribution of the sociodemographic data among women. Approximately half (54\%) of the teachers were aged less than 40 years, while one fifth $(21.6 \%)$ were between 40 and 45 years, $17.2 \%$ were between 45 and 50 years, and only $7.2 \%$ were over 50 years. Regarding the teachers' educational level, approximately half $(54 \%)$ of them had university level education, $34.5 \%$ had diploma, only $8.9 \%$ had up to secondary school education while $2.9 \%$ had other education as well as educational institute certificate. Regarding marital status, the majority of teachers were married $(81.6 \%), 9.5 \%$ were single, $6 \%$ were divorced and only $2.9 \%$ were widowed. Family size of the teachers distributed between 2-4, 5-6 and more than six at 41.7\%, 35.1\% and $23.3 \%$ respectively. Nearly half of the teachers' family income $(55.5 \%)$ were over $10,000 \mathrm{SAR}$, one-third $(35.3 \%)$ between 5,000 to $10,000 \mathrm{SAR}$, and only $8.9 \%$ of teachers less than 5,000 SAR.

Table 2 shows obstetric history among female teachers in
Dawadmi city. Most of the female teachers (82.4\%) experienced menarche below 14 years and have regular menstruation $(79.9 \%)$. In addition, only $16.1 \%$ did not get pregnant and $15.5 \%$ had no parity. Furthermore, $66.1 \%$ did not complain of chronic diseases, while the others had different kinds of such diseases including cardiac disease $(2.3 \%)$, diabetes $(6 \%)$, hypertension $(10.6 \%)$, thyroid disease $(12.1 \%)$ and other diseases such as chronic inflammations $(2.9 \%)$. Note that thyroid disease appeared more frequently than other diseases.

Table 1. Distribution of the sample according to their socio-demographic characteristics

\begin{tabular}{|c|c|c|}
\hline Socio-demographic data & N (348) & $\%$ \\
\hline \multicolumn{3}{|l|}{ Age } \\
\hline $35<40$ & 188 & 54.0 \\
\hline $40<45$ & 75 & 21.6 \\
\hline $45<50$ & 60 & 17.2 \\
\hline$\geq 50$ & 25 & 7.2 \\
\hline \multicolumn{3}{|l|}{ Level of education } \\
\hline Secondary school & 31 & 8.9 \\
\hline Diploma & 120 & 34.5 \\
\hline University & 187 & 53.7 \\
\hline Other & 10 & 2.9 \\
\hline \multicolumn{3}{|l|}{ Marital status } \\
\hline Single & 33 & 9.5 \\
\hline Married & 284 & 81.6 \\
\hline Divorced & 21 & 6.0 \\
\hline Widow & 10 & 2.9 \\
\hline \multicolumn{3}{|l|}{ Family size } \\
\hline $2-4$ & 145 & 41.7 \\
\hline $5-6$ & 122 & 35.1 \\
\hline 6 and more & 81 & 23.3 \\
\hline \multicolumn{3}{|c|}{ Income of the family (Saudi Riyal) } \\
\hline Less than 5,000 & 31 & 8.9 \\
\hline $5,000-10,000$ & 123 & 35.3 \\
\hline 10,000 and more & 193 & 55.5 \\
\hline
\end{tabular}

Table 3 shows the scoring of knowledge about menopause among female teachers in Dawadmi city. It was found that $58.9 \%$ of teachers had poor level of knowledge, $30.7 \%$ moderate knowledge and only $10.3 \%$ of teachers had good and excellent knowledge levels ( $8 \%$ and $2.3 \%$ respectively). According to the data, the majority of teachers $(74.7 \%)$ had well recognized the concept of menopause, while half of them $(50.9 \%)$ knew the exact age of menopause. Furthermore, causes of menopause and time of appearance of symptoms were lesser known at $34.8 \%$ and $15.2 \%$ of teachers respectively. Table 3 also shows the distribution of knowledge of female teachers regarding signs and symptom of 
menopause, which were grouped into four-main categories (physical symptoms, psychological symptoms, social symptoms and symptoms associated with the reproductive system). Physical symptoms were represented by 10 items. In general, nearly half $(46 \%)$ of female teachers knew these physical symptoms. Psychological symptoms were represented by six items, $50 \%$ recognized them. In contrast, social symptoms were only identified by $36.9 \%$ of teachers in general. Lastly, symptoms associated with the reproductive system were generally recognized by $34.5 \%$ of female teachers.

Table 2. Distribution of sample according to obstetric and medical history

\begin{tabular}{|c|c|c|}
\hline Obstetric history & N (348) & $\%$ \\
\hline \multicolumn{3}{|l|}{ Age at menarche } \\
\hline Before 12 years & 68 & 19.5 \\
\hline $12-14$ years & 219 & 62.9 \\
\hline $15-16$ years & 49 & 14.1 \\
\hline More than 16 years & 12 & 3.4 \\
\hline \multicolumn{3}{|c|}{ Menstruation regular } \\
\hline Yes & 278 & 79.9 \\
\hline No & 70 & 20.1 \\
\hline \multicolumn{3}{|c|}{ Number of pregnancy } \\
\hline No pregnancy & 56 & 16.1 \\
\hline 1-3 pregnancies & 114 & 32.8 \\
\hline 4-5 pregnancies & 117 & 33.6 \\
\hline 6 and more & 61 & 17.5 \\
\hline \multicolumn{3}{|l|}{ Number of parity } \\
\hline No parity & 54 & 15.5 \\
\hline Para 1 & 24 & 6.9 \\
\hline Para 2-3 & 121 & 34.8 \\
\hline Para 4-5 & 96 & 27.6 \\
\hline Para 6 and above & 53 & 15.2 \\
\hline \multicolumn{3}{|l|}{ Chronic diseases } \\
\hline Cardiac Disease & 8 & 2.3 \\
\hline Diabetes & 21 & 6.0 \\
\hline Hypertension & 37 & 10.6 \\
\hline Thyroid Disease & 42 & 12.1 \\
\hline Other & 10 & 2.9 \\
\hline None & 230 & 66.1 \\
\hline
\end{tabular}

Table 3. Distribution of total score of knowledge about menopausal period of the study sample

\begin{tabular}{lll}
\hline Levels of knowledge about menopause & $\mathbf{N}(\mathbf{3 4 8 )}$ & $\mathbf{\%}$ \\
\hline Poor knowledge & 205 & 58.9 \\
Moderate knowledge & 107 & 30.7 \\
Good knowledge & 28 & 8.0 \\
Excellent knowledge & 8 & 2.3 \\
Total & 348 & 100.0 \\
\hline
\end{tabular}

Table 4 shows the total mean score and standard deviation (SD) of the Health Promoting Behaviors and the Subdomains in female teachers. Health Responsibility showed a mean (20.43 \pm 6.404$)$, which considered medium level of health promotion, Physical Activity showed a mean of $15.86 \pm 5.648$ which is considered low level, Nutrition reported a mean of $21.74 \pm 5.441$ which is considered medium, Spiritual Growth has a mean of $25.30 \pm 6.933$ and is considered medium, Interpersonal Relations has a mean of $25.39 \pm$ 7.135 which is medium and Stress Management has a mean of $20.81 \pm 5.311$ which shows a medium level of health promotion behavior. Total Health Promoting Behaviors showed a mean of $130.05 \pm 27.555$ which is considered medium in general.

Table 4. Total mean and standard deviation (SD) of Health Promoting Behaviors and the Sub-domains in the study sample

\begin{tabular}{llll}
\hline & Mean & S.D. & Level of practice \\
\hline Health Responsibility & 20.43 & 6.404 & medium \\
Physical Activity & 15.86 & 5.648 & low \\
Nutrition & 21.74 & 5.441 & medium \\
Spiritual Growth & 25.30 & 6.933 & medium \\
Interpersonal Relations & 25.39 & 7.135 & medium \\
Stress Management & 20.81 & 5.311 & medium \\
Total & 130.05 & 27.555 & medium \\
$\begin{array}{l}\text { Note. The mean of total behaviors was 130.05 which represents a medium level } \\
\text { of practice (104-155). }\end{array}$ & & &
\end{tabular}

Table 5 shows the relationship between total knowledge level and total behavior level. The results show a significant correlation $(p=.000)$. To find out the direction of the function between variables, Spearman's rho test was performed. Correlation coefficient for medical history vs knowledge score is 0.122 when Sig. $=0.022$. This result suggests that the variables have a positive correlation.

Table 6 shows the association between socio-demographic variable of female teachers and their level of knowledge, in addition to frequencies of each category item with regard to total knowledge level. In general, socio-demographic factors did not show significant correlations according to the Pearson Chi-Square test. ANOVA test did not show significant correlation for total knowledge level as well. However, some of the knowledge categories showed significant correlations with socio-demographic data as well as knowledge about menopause which correlate significantly with weight in the last year $(\mathrm{F}=3.662, p=.013)$. In addition, knowledge about the age women usually attain menopause also correlates significantly with weight during the last year $(\mathrm{F}=.3064, p=$ .048). Furthermore, knowledge about physical symptoms of menopause correlates significantly with family income ( $\mathrm{F}$ 
$=.2976, p=.001)$. In addition, knowledge about strategies applied to reduce the symptoms of menopause also correlates significantly with weight during the last year $(\mathrm{F}=.3194, p$ $=.024)$. Knowledge about strategies applied to reduce the symptoms of menopause (Nutrition) also correlates significantly with family income $(\mathrm{F}=3.271, p=.021)$ and family size $(\mathrm{F}=5.899, p=.001)$. In addition, knowledge about strategies applied to reduce the symptoms of menopause
(Overcome weight gain) correlates significantly with family size $(\mathrm{F}=3.622, p=.013)$. Knowledge about strategies applied to reduce the symptoms of menopause (Overcome feeling of warmth) correlates significantly with family income $(\mathrm{F}=3.848, p=.004)$. Knowledge about strategies applied to reduce the symptom of menopause (Overcome headache) correlates significantly with family income $(\mathrm{F}=$ $3.126, p=.026)$ and family size $(\mathrm{F}=3.617, p=.013)$.

Table 5. Association between health promoting behavior and knowledge about menopause

\begin{tabular}{|c|c|c|c|c|c|}
\hline \multirow{2}{*}{ Total knowledge score } & \multicolumn{3}{|c|}{ Total health promoting lifestyle behavior score } & \multirow{2}{*}{ Pearson $\chi^{2}$} & \multirow{2}{*}{$p$} \\
\hline & Low & Moderate & High & & \\
\hline Poor knowledge & 29 & 152 & 23 & & \\
\hline Moderate knowledge & 21 & 73 & 13 & & \\
\hline Good knowledge & 1 & 12 & 15 & $37.951 *$ & .000 \\
\hline Excellent knowledge & 1 & 5 & 2 & & \\
\hline Total & 52 & 242 & 53 & & \\
\hline
\end{tabular}

${ }^{*} p<.05$

Table 6. Correlation between socio-demographic variables of the sample and their level of knowledge

\begin{tabular}{|c|c|c|c|c|c|c|}
\hline Socio-demographic & Poor & Moderate & Good & Excellent & Pearson $\chi^{2}$ & $p$ \\
\hline \multicolumn{7}{|l|}{ Age } \\
\hline $35<40$ & 112 & 55 & 16 & 5 & \multirow{4}{*}{9.096} & \multirow{4}{*}{.429} \\
\hline $40<45$ & 42 & 26 & 5 & 2 & & \\
\hline $45<50$ & 36 & 21 & 2 & 1 & & \\
\hline$\geq 50$ & 15 & 5 & 5 & 0 & & \\
\hline \multicolumn{7}{|l|}{ Level of education } \\
\hline Secondary school & 21 & 7 & 2 & 1 & \multirow{4}{*}{5.188} & \multirow{4}{*}{.818} \\
\hline Diploma & 68 & 40 & 9 & 3 & & \\
\hline University & 110 & 58 & 16 & 3 & & \\
\hline Other & 6 & 2 & 1 & 1 & & \\
\hline \multicolumn{7}{|l|}{ Marital status } \\
\hline Single & 21 & 9 & 1 & 2 & \multirow{4}{*}{13.605} & \multirow{4}{*}{.137} \\
\hline Married & 169 & 88 & 22 & 5 & & \\
\hline Divorced & 8 & 7 & 5 & 1 & & \\
\hline Widow & 7 & 3 & 0 & 0 & & \\
\hline \multicolumn{7}{|l|}{ Family size } \\
\hline $2-4$ & 84 & 44 & 13 & 4 & \multirow{3}{*}{2.039} & \multirow{3}{*}{.916} \\
\hline $5-6$ & 70 & 41 & 8 & 3 & & \\
\hline 6 and more & 51 & 22 & 7 & 1 & & \\
\hline \multicolumn{7}{|c|}{ Income of the family ( Saudi Riyal) } \\
\hline Less than 5,000 & 14 & 10 & 5 & 2 & \multirow{4}{*}{10.437} & \multirow{3}{*}{.107} \\
\hline $5,000-10,000$ & 74 & 35 & 13 & 1 & & \\
\hline 10,000 and more & 116 & 62 & 10 & 5 & & \\
\hline \multicolumn{6}{|c|}{ Weight during last year } & \\
\hline Normal & 90 & 50 & 13 & 7 & \multirow{4}{*}{9.922} & \multirow{4}{*}{.357} \\
\hline Above the normal & 83 & 47 & 11 & 1 & & \\
\hline Less than normal & 7 & 3 & 2 & 0 & & \\
\hline I don't know & 25 & 7 & 2 & 0 & & \\
\hline
\end{tabular}


Table 7 shows the association between socio-demographic level $(p=.014)$ using Pearson Chi-Square test. However, variables of female teachers and their health promoting behaviors about menopause, in addition to frequencies of each item with regard to total health promoting behaviors level. ANOVA test was also done for total behaviors level, which also showed significant correlation with age $(\mathrm{F}=1.358, p=$ Only weight during last year correlated with total behaviors

$.028)$ and weight during last year $(\mathrm{F}=1.303, p=.049)$.

Table 7. Correlation between socio-demographic variables of the sample and their health promoting behaviors in menopause

\begin{tabular}{|c|c|c|c|c|c|}
\hline Socio-demographic & Low & Moderate & High & Pearson $\chi^{2}$ & $p$ \\
\hline \multicolumn{6}{|l|}{ Age } \\
\hline $35<40$ & 25 & 127 & 35 & \multirow{4}{*}{8.288} & \multirow{4}{*}{.218} \\
\hline $40<45$ & 16 & 52 & 7 & & \\
\hline $45<50$ & 7 & 47 & 6 & & \\
\hline$\geq 50$ & 4 & 16 & 5 & & \\
\hline \multicolumn{6}{|l|}{ Level of education } \\
\hline Secondary school & 3 & 26 & 2 & \multirow{4}{*}{3.810} & \multirow{4}{*}{.702} \\
\hline Diploma & 19 & 82 & 19 & & \\
\hline University & 28 & 127 & 31 & & \\
\hline Other & 2 & 7 & 1 & & \\
\hline \multicolumn{6}{|l|}{ Marital status } \\
\hline Single & 7 & 26 & 0 & \multirow{4}{*}{9.452} & \multirow{4}{*}{.150} \\
\hline Married & 40 & 193 & 50 & & \\
\hline Divorced & 3 & 15 & 3 & & \\
\hline Widow & 2 & 8 & 0 & & \\
\hline \multicolumn{6}{|l|}{ Family size } \\
\hline $2-4$ & 23 & 101 & 21 & \multirow{3}{*}{5.946} & \multirow{3}{*}{.203} \\
\hline $5-6$ & 15 & 81 & 25 & & \\
\hline 6 and more & 14 & 60 & 7 & & \\
\hline \multicolumn{6}{|l|}{ Income of the family } \\
\hline Less than 5,000 & 3 & 23 & 5 & \multirow{4}{*}{2.181} & \multirow{3}{*}{.703} \\
\hline $5,000-10,000$ & 22 & 81 & 20 & & \\
\hline 10,000 and more & 26 & 138 & 28 & & \\
\hline \multicolumn{5}{|c|}{ Weight during last year } & \\
\hline Normal & 29 & 109 & 22 & \multirow{4}{*}{$29.088^{*}$} & \multirow{4}{*}{.014} \\
\hline Above the normal & 17 & 104 & 20 & & \\
\hline Less than normal & 0 & 9 & 3 & & \\
\hline I don't know & 6 & 20 & 8 & & \\
\hline
\end{tabular}

Health promoting behaviours in menopause are composed of different behavioural categories namely Health Responsibility, Physical Activity, Nutrition, Spiritual Growth, Interpersonal Relations and Stress Management. Regarding behaviours in health responsibility, there are significant correlations between it with age $(\mathrm{F}=1.556, p=.041)$ and family size $(\mathrm{F}=2.126, p=.001)$. In addition, behaviours in nutrition correlate significantly with marital status $(\mathrm{F}=1.942, p$ $=.005)$.

\section{Discussion}

Most women attain menopause without having adequate knowledge about its consequences. In some studies, it is found that women who have better information about menopause experience fewer consequences of the phenomenons. Therefore, those who have adequate knowledge and information of symptoms and complications of menopause are able to better cope with it and take appropriate treatment. As menopause is a natural phenomenon, every woman will face menopause but awareness regarding its problems faced during menopause needs to be addressed. ${ }^{[20,21]}$

In the present study, $41 \%$ of female teachers had moderate to excellent knowledge of menopause in general, while $59 \%$ had poor knowledge regarding menopause. Similar findings were observed in a study done in Lumbini Medical College 
Teaching Hospital, ${ }^{[22]}$ showing $63.4 \%$ of respondents with poor knowledge, $33.8 \%$ had fair and only $2.8 \%$ had good level of knowledge regarding menopause. Also a study done in India ${ }^{[23]}$ showed similar results when knowledge regarding signs and symptoms of menopause were assessed. They found that $44 \%$ of respondents range from moderate to adequate knowledge while $56 \%$ had inadequate knowledge.

These results reflect those of the teachers. As they play different roles as teachers, mothers and role models in the Dawadmi community. There is a need for a structured health education program based on their and the community's needs. Several studies done in Egypt, Turkey, India, and Korea to assess the effectiveness of education programs in menopausal and premenopausal women showed a significant increase in the knowledge of women regarding menopausal symptoms and its management. ${ }^{[24-27]}$

In the present study, the majority of teachers received their information about menopause from more than one source such as TV, magazine and family and friends. Only 3\% received their information from physicians and health workers. The finding is in line with the study done among midlife school teachers in India ${ }^{[28]}$ that showed $62 \%$ obtained their information from friends and relatives and only $10 \%$ from doctors.

The reason behind the poor knowledge may be due to inappropriate sources of information. Therefore, it is important for medical professionals to teach their clients self coping strategies especially in the effects of menopause. There should also be a collaboration between the Ministry of Health, Ministry of Education, and Ministry of Culture and Information regarding health education programs on women's health especially for women in Saudi Arabia.

Women with insufficient or incorrect knowledge about menopause need to be encouraged to participate in health management activities for better knowledge and care. Knowledge of menopause was found to be crucial in improving ability to manage menopause.

Health promotion is one of the ways to promote quality of life and eventually reduce health expenses. Complications associated with post menopause can be reduced or managed if women adopt health promoting behavior which include self actualization, self responsibility, exercise, nutrition, stress management, and interpersonal relationship aspects. ${ }^{[29]}$

An initial objective of this study is to assess health promoting behavior during the premenopausal period. The total mean score of health promoting lifestyle behaviors of our participants is reported in the moderate range which is consistent with other studies conducted on Iranian middle-aged women, another Taiwan study, and a study done in menopausal women in Iran. ${ }^{[19,30,31]}$ It would seem that women need greater attention from policy makers and health care services to establish health promotion training programs tailored to them. An interesting result in the present study was that the respondents scored a higher mean for interpersonal relations (25.39), consistent with the result of a study in Turkey. ${ }^{[32]}$ These results might be due to the subjects are colleagues, therefore they build good relationships with others. These results also reflect the Saudi Arabian culture in giving support to each other during ease and difficult times.

Spiritual health is considered as one of the most essential aspects of human health. Spirituality gives people meaning and direction in life. It provides a harmonious and integrated relationship between the internal forces and it is distinguished with characteristics of stability in life, peace, coordination and a feeling of closeness with the self, God, society and environment. ${ }^{[33,34]}$ In this study we reached a high mean score (25.30) which is similar to the Turkish nurses and menopausal Iranian women of aforementioned studies. ${ }^{[19,35]}$ This is also a higher result than the Taiwan study. ${ }^{[31]}$ This can be due to the importance rule of the Islamic religion in Saudi Arabia.

In this study female teachers have the lowest score in physical activity with mean score 15.86 . This is similar to the study by Enjezab (2012) that was conducted on middle-aged Iranian women. The study was also conducted among nurses working in Ankara University Medical Faculty Hospital in Turkey. ${ }^{[30,35]}$ It is clear that physical activity is necessary for all individuals especially middle-aged women and inadequate physical activity causes cardiovascular diseases, diabetes and osteoporosis. ${ }^{[36]}$ In this study it was found that $41 \%$ of teachers were above the normal weight which they attributed to a lack of physical activity. The reason for the lack of physical activity in the Dawadmi society may be because of the lack of women's positive belief and attitude towards physical activity. In review of the study done by Oguma and Shinoda-Tagawa in $2014,{ }^{[37]}$ the results indicate that physical activity is associated with a significant reduction of chronic heart diseases, stroke and hypertension among women. The significant role of exercise in decreasing the risk of diabetes mellitus was found in a recent paper by Grace et al. ${ }^{[38]}$

Previous studies showed that physical activity has positive effects on alleviating menopausal symptoms. ${ }^{[39,40]}$ Therefore, there is a need for interventional programs for premenopausal women on the importance of exercise to reduce the chances of cardiovascular disease, osteoporosis, type 2 diabetes and improve the state of their health.

Health responsibility scored second in this study, after phys- 
ical exercise with a mean of 20.43 and this result is in line with previous middle age Iranian women and the Turkish nurses. ${ }^{[30]}$ This may be because individuals did not consider control of their health as a necessity. Naturally, an individual would not do anything to promote his/her health unless he/she has a problem with it. ${ }^{[41]}$ Being responsible for one's health results in an improvement of the health status and also prevention of disease.

Therefore, it is essential for nurses to educate patients on this. Stress management comes after health responsibility with a moderate mean score of 20.81. This result matches with the urban Iranian women study. ${ }^{[19]}$ Physical health outcomes are related to stress including hypertension as mentioned by Din-Dzietham et al. ${ }^{[42]}$ Furthermore, biological responses resulting from stress are possible contributors to the development of a number of physiological risk factors for cardiovascular disease, stroke, type 2 diabetes and several cancers. ${ }^{[43]}$ Stress-related health problems are common among working people. For this reason, nurses should educate female teachers about work stress and its causes as well as strategies to reduce stress.

Next, nutrition scored a mean of 21.74 which is similar to the result of the Turkish nurses. ${ }^{[35]}$ More often than not, women attain menopause unprepared to cope with the changes of this period of life, and an insufficient knowledge of dietary habits may lead to the oversupply or lack of nutrients. A healthy diet should be balanced, safe and protective. A balanced diet prevents obesity and diseases that may be caught due to a lack of energy and essential nutrients. Nutrition, according to the standards and recommendations of the WHO results in better physiological nutritional of menopausal women, result in a reduction of morbidity and negative symptoms of menopause.

This study showed a significant correlation for total health behaviors with age ( $\mathrm{F}=1.358, p=.028)$. These results are consistent with the study of middle age Iranian women. ${ }^{[30]}$ This study also shows a significant correlation for total health behaviors and weight during last year for teachers $(\mathrm{F}=1.303$, $p=.049)$. A similar study of postmenopausal Iranian women also showed a significant relationship between the whole score of health promoting behavior and body mass index of women and their level of education. ${ }^{[19]}$ However, this study didn't show any significant correlations with education.

There are also significant correlations between the subdomain of health responsibility with age $(\mathrm{F}=1.556, p=$ $.041)$ and family size $(\mathrm{F}=2.126, p=.001)$.

In addition, nutrition correlated significantly with marital status $(\mathrm{F}=1.942, p=.005)$. These results are in agreement with those obtained from postmenopausal Iranian women. ${ }^{[5]}$

In general, female teachers in Dawadmi city are desirable in mental health dimensions of HPBs such as stress management, interpersonal relations and spiritual growth. However, their behaviors in the physical health dimensions (physical activity, health responsibility and nutrition) we extremely undesirable and may negatively impact their physical health later in life. In order to smoothen the aging process, improvements to their lifestyle needs to be made before early middle age. Moreover, it is critical for health professionals to be aware of the special needs of middle-aged women in order to help them better cope with the next stage in their lives. Encouraging HPBs in middle-aged women should be a priority for maternity nurses. Through nurses, health promotion can be done in empowering and motivating menopausal women to be better self-managers and reinforce positive attitudes. In addition, a better understanding of menopause in general can help nurses to better intervene in their clients with best nursing practices pertaining the experienced symptoms. Through nurses, women are also able to get up to date knowledge to enable them to cope. Nurses have to remember that all the menopausal assessments should be individualized as well as in cooperation with holistic care. One of the most important duties of health center staffs especially maternity nurses and midwives who are responsible for taking care of postmenopausal women is to explain health promoting behaviors in addition to designing and performing educational interventional plans to create motivation towards a healthy lifestyle.

\section{Implication of the study}

This study provides a first step in better understanding of assessment knowledge and health promoting behavior among teachers in Dawadmi city.

The assessment of menopause knowledge and health promoting behavior may be useful for understanding why women hesitate to seek medical care for menopausal symptom and develop intervention to improve coping behavior of women with menopausal symptoms in the community. Nurses, healthcare practitioners, and policy makers should educate teachers in coping strategies for menopausal symptoms offer access to information and resources as well as counselling activities to prepare the teachers to help themselves, students and the community to create a positive attitude and healthy perception.

\section{Conclusion}

The findings of this study showed that female teachers in Dawadmi city generally had poor knowledge regarding menopause. Overall, the socio-demographic variable of fe- 
male teachers did not show significant correlation with the total level of knowledge. The level of health promoting behavior during the premenopausal period of female teachers was reported to be in the moderate range. Subjects scored fairly lowly on physical exercise. In addition, the study showed significant correlation for total health promotion behaviors with age.

\section{Recommendations}

According to the results of the present study, it is important to give the opportunity to teachers to attend health promotion training to help them increase their perception of the effec- tiveness and accountability of health promotion programs as teacher training is a key factor for the development of health promotion issues. Saudi Arabia, like other Gulf nations, have experienced both directly and indirectly major social upheavals. Therefore, it is in need of more research on the effects of culture on knowledge and health promotion practices. This research must be replicated in other cities to address the problem with women in their pre-peri-post menopausal period.

\section{CONFLiCTS OF INTEREST Disclosure}

The authors declare that there is no conflict of interest.

\section{REFERENCES}

[1] Statistics Korea. 2012 life tables for Korea. Daejeon: Statistics Korea, 2012. [Cited by 2014 March 20]. Available from: http: //kosis.kr/statisticsList/statisticsList_01List

[2] Press D. Menopausal symptoms and quality of life among Saudi women visiting primary care clinics in Riyadh, Saudi Arabia. 2015; 645-53.

[3] Rahman SASA, Zainudin SR, Mun VLK. Assessment of menopausal symptoms using modified Menopause Rating Scale (MRS) among middle age women in Kuching, Sarawak, Malaysia. Asia Pac Fam Med. 2010; 9(1): 5. PMid:20175928 https ://doi.org/10.118 6/1447-056X-9-5

[4] Dickey RA, Janick JJ. Lifestyle modifications in the prevention and treatment of hypertension. Endocr Pract. 2001; 7(5): 392-9. PMid:11585378 https://doi.org/10.4158/EP.7.5.392

[5] Sehhatie F, Mirghafourvand M, Momeni K. Health promoting behaviors among postmenopausal women in Langroud city, Iran. Int J Women's Heal Reprod Sci. 2015; 3(3): 158-62. https://doi .or g/10.15296/ijwhr.2015.33

[6] Population estimates General Organization for Statistics. cited 2018 Apr 12. Available from: https://www.stats.gov.sa/ar/43

[7] Alquaiz AM, Siddiqui AR, Qureshi RH, et al. Women Health in Saudi Arabia: A review of non-communicable diseases and their risk factors. Pakistan J Med Sci. 2014 Mar; 30(2): 422-31.

[8] Borzou SR, Zonoori S, Falahinia GH, et al. The effect of education of health-promoting behaviors on lifestyle in hemodialysis patients. Int J Med Res Heal Sci. 2016; 5(6): 214-20.

[9] Bahadır Z, Certel Z, Topuz R. a Research on Healthy Living Behaviors of Archery Coaches and Boxing Coaches. Int J Sci Cult Sport. 2014; 2(5): 88-96.

[10] Abo E, Elhossiny E, El-zeftawy AMA. Knowledge of Women in Reproductive Age about Menopausal Problems and Preventive Health Behaviors in Tanta City, Al-Gharbyia Governorate, Egypt. 2015; 4(3): 51-63.

[11] Noroozi E, Dolatabadi NK, Eslami AA, et al. Knowledge and attitude toward menopause phenomenon among women aged $40-45$ years. J Educ Health Promot. 2013; 2(May): 25.

[12] Golyan Tehrani S, Mir Mohammad Ali M, Mahmoudi M, et al. Study of quality of life and its patterns in different stages of menopause for women in Tehran. J Hayat. 2002; 8(3): 33-41.
[13] Sherman S. Defining the menopausal transition. cited $2018 \mathrm{Apr}$ 13. Available from: http://www.amjmed.com/article/S0002 $-9343(05) 01039-9 / p d f$

[14] Stages of Menopause. cited 2018 Apr 13. Available from: http: //www.acsm.org/public-information/articles/20 16/10/07/stages-of-menopause

[15] Pender NJ. Health Promotion Model Manual. 2011. Available from: http://hdl.handle.net/2027.42/85350

[16] Sample Size Formulas for our Sample Size Calculator-Creative Research Systems. [cited 2018 Feb 4]. Available from: https: //www. surveysystem.com/sample-size-formula.htm

[17] Rukumani J. Knowledge on Menopause among Pre Menopausal Women at Selected Area of Puducherry, India. 2015; 3(8): 1-3.

[18] Walker SN, Sechrist KR, Pender NJ. The health-promoting lifestyle profile: development and psychometric characteristics. Nurs Res. 1987; 36(2): 76-81.

[19] Asrami FS, Hamzehgardeshi Z, Shahhosseini Z. Health Promoting Lifestyle Behaviors in Menopausal Women: A Cross-Sectional Study. 2016; 8(8): 128-34.

[20] Fallahzadeh H, Hossienzadeh M, Yazdani F, et al. Knowledge of reproductive physiology and hormone therapy in 40-60 year old women: a population-based study in Yazd, Iran. Iran J Reprod Med. 2012 Jul; 10(4): 383-90.

[21] Mohamed HA. Improving women's practices for reducing the severity of menopausal symptoms. 2016; 6(4).

[22] Paudyal P, Nepal M. Knowledge on Perimenopausal Symptoms among Women Attending Lumbini Medical College Teaching Hospital. J Lumbini Med Coll. 2014; 2(2): 41-4. https ://doi .org/10 .22502/jlmc.v2i2.56

[23] Barathi1 SK. Assessment of Knowledge on Signs and Symptoms of Menopause among Premenopausal Women. IOSR J Nurs Heal Sci. 2014; 3(2): 33-7. https : //doi .org/10.9790/1959-03233337

[24] Orabi EE. Effect of Health Education Intervention on Knowledge, and attitude regarding Menopausal Period among Premenopausal Female Employees. Heal Eduction Interv Knowl Egypt J Community Med. 2017; 35(3).

[25] Tortumluoglu G, Erci B. The Effectiveness of Planned Health Education Given to Climacteric Women on Menopausal Symptoms, Menopausal Attitude and Health Behaviours. Int J Hum Sci. 2004; $1-22$.

[26] Nair NP, Kumar V. A study to assess the effectiveness of structured teaching programme on the knowledge of women regarding 
menopausal changes in selected rural areas of Mysore. Asian J Nurs Educ Res. 2015; 5(3): 385. https : //doi.org/10.5958/2349-2 996.2015 .00077 .4

[27] Kwak EK, Park HS, Kang NM. Menopause Knowledge, Attitude, Symptom and Management among Midlife Employed Women. J menopausal Med. 2014 Dec; 20(3): 118-25. PMid:25580423 https: //doi.org/10.6118/jmm.2014.20.3.118

[28] Sultan S, Sharma A, Jain NK. Knowledge, attitude and practices about menopause and menopausal symptoms among midlife school teachers. 2017; 6(12): 1-5.

[29] Sorour AS, Kamel WW, Mohammed E, et al. Health promoting lifestyle behaviors and related risk factors among female employees in Zagazig city. 2014; 4(5).

[30] Enjezab B, Farajzadegan Z, Taleghani F, et al. Health Promoting Behaviors in a Population-based Sample of Middle-aged Women and its Relevant Factors in Yazd, Iran. Int J Prev Med. 2012 Mar; 3(Suppl 1): S191-8.

[31] Lee FH, Wang HH, Lee FH, et al. A PRELIMINARY STUDY OF A HEALTH-PROMOTING LIFESTYLE AMONG SOUTHEAST ASIAN WOMEN IN TAIWAN. Kaohsiung J Med Sci. 2005; 21(3): 114-20. https://doi.org/10.1016/S1607-551X (09) 7 0287-6

[32] Beser A, Bahar Z, Büyükkaya D. Health Promoting Behaviors and Factors related to Lifestyle among Turkish Workers and Occupational Health Nurses' Responsibilities in their Health Promoting Activiti. Ind Health. 2007; 45: 151-9. https://doi.org/10.2486/indh ealth. 45.151

[33] Adegbola M. Spirituality, Self-Efficacy, and Quality of Life among Adults with Sickle Cell Disease. South Online J Nurs Res. 2011 Apr; 11(1).

[34] Rezaei M, Adib-Hajbaghery M, Seyedfatemi N, et al. Prayer in Iranian cancer patients undergoing chemotherapy. Complement Ther Clin Pract. 2008 May; 14(2): 90-7.
[35] Kirag N, Ocaktan EM. Analysis of health promoting lifestyle behaviors and associated factors among nurses at a university hospital in Turkey. Saudi Med J. 2013 Oct [cited 2018 Jan 2]; 34(10): 1062-7.

[36] Ekelund U, Besson H, Luan J, et al. Physical activity and gain in abdominal adiposity and body weight: prospective cohort study in 288,498 men and women. Am J Clin Nutr. 2011 Apr 1; 93(4): 826-35.

[37] Oguma Y, Shinoda-Tagawa T. Physical activity decreases cardiovascular disease risk in women. Am J Prev Med. 2004 Jun; 26(5): 407-18. PMid:15165657 https://doi .org/10.1016/j . amepre .2004 .02 .007

[38] Grace SL, Barry-Bianchi S, Stewart DE, et al. Physical Activity Behavior, Motivational Readiness and Self-Efficacy among Ontarians with Cardiovascular Disease and Diabetes. J Behav Med. 2007 Jan 31; 30(1): 21-9.

[39] Daley A, Thomas A, Roalfe A, et al. The effectiveness of exercise as treatment for vasomotor menopausal symptoms: randomised controlled trial. BJOG An Int J Obstet Gynaecol. 2015 Mar; 122(4): 565-75.

[40] Sternfeld B, Guthrie KA, Ensrud KE, et al. Efficacy of exercise for menopausal symptoms. Menopause. 2013 Aug; 21(4): 1.

[41] Sullivan PW, Morrato EH, Ghushchyan V, et al. Obesity, inactivity, and the prevalence of diabetes and diabetes-related cardiovascular comorbidities in the U.S., 2000-2002. Diabetes Care. 2005 July; 28(7): 1599-603.

[42] Din-Dzietham R, Nembhard WN, Collins R, et al. Perceived stress following race-based discrimination at work is associated with hypertension in African-Americans. The metro Atlanta heart disease study, 1999-2001. Soc Sci Med. 2004 Feb [cited 2018 Jan 4]; 58(3): 449-61.

[43] Minimizing the Risks of Cardiovascular DiseaseCanada.ca. cited 2018 Jan 4. Available from: https: //www.canada.ca/en/public-health/services/chron ic-diseases/cardiovascular-disease/minimizing-ris ks-cardiovascular-disease.html 